Revue de droit comparé du travail et de la sécurité sociale

\title{
L'emploi informel au Mexique et en Amérique centrale : un phénomène complexe
}

\section{Alfredo Sánchez-Castañeda}

\section{(2) OpenEdition}

\section{Journals}

Édition électronique

URL : https://journals.openedition.org/rdctss/364

DOI : $10.4000 /$ rdctss.364

ISSN : 2262-9815

Éditeur

Centre de droit comparé du travail et de la sécurité sociale

Édition imprimée

Date de publication : 1 septembre 2017

Pagination : $90-115$

ISSN : 2117-4350

Référence électronique

Alfredo Sánchez-Castañeda, «L'emploi informel au Mexique et en Amérique centrale : un phénomène complexe », Revue de droit comparé du travail et de la sécurité sociale [En ligne], 3 | 2017, mis en ligne le 01 novembre 2018, consulté le 04 décembre 2021. URL : http://journals.openedition.org/rdctss/364 DOI : https://doi.org/10.4000/rdctss.364

\section{(c) (i) (2) $९$}

Revue de droit comparé du travail et de la sécurité sociale est mise à disposition selon les termes de la Licence Creative Commons Attribution - Pas d'Utilisation Commerciale - Pas de Modification 4.0 International. 


\section{L'emplol informel au MeXiQue et eN AMÉRIQUE CENTRALE : Un PHÉNOMĖNE COMPLEXE}

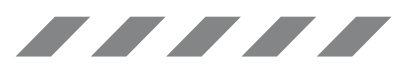

\section{ABSTRACT}

The possibility of a joint study of informal employment in Mexico and Central America is proposed. The first part, shows how the informal economy is a structural phenomenon with a plurality of jobs, classified according to the ILO's international instruments. Subsequently, we analyze the multiplicity of jobs, which intersect repeatedly, in the countries studied and the effects of public policies to formalize informal employment. We note the low effectiveness of these policies.

\section{KEYWORDS : Informal Economy, informal employment, Mexico, central America.}

\section{RÉSUMÉ}

Cet article propose d'étudier l'emploi informel au Mexique et en Amérique centrale. La première partie montre que l'économie informelle est un phénomène structurel, touchant de nombreux emplois, que l'on classe à partir des instruments internationaux de l'OIT. Par la suite, nous analyserons la diversité des emplois concernés dans les pays étudiés et les effets des politiques publiques visant à formaliser l'emploi informel. Le constat s'impose de la faible efficacité de ces politiques.

MOTS CLÉS : ÉConomie informelle, emploi informel, Mexique, Amérique centrale. 


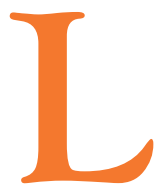

e droit joue un rôle fondamental dans le débat sur l'informalité. Il serait inexact de considérer que la loi ignore le travail informel. Une occupation professionnelle, même de cette nature, a forcément des conséquences juridiques. En ce sens, s'il y a des relations de travail dans le secteur informel, elles doivent être reconnues sans aucune ambiguïté, en particulier quand il s'agit de travailleurs subordonnés déguisés en travailleurs indépendants ou en entrepreneurs. Cependant, il est également important de souligner la complexité de l'emploi informel, tel qu'il est appréhendé par les instruments internationaux et tel qu'il se manifeste dans les pays analysés.

Il faut préciser préalablement, que l'Amérique latine se compose de pays très différents, qui se distinguent aussi bien par la langue, que par leurs traditions, culture et origines. Peut-on englober dans cette étude l'Amérique ${ }^{01}$ centrale (Guatemala, Belize, Honduras, Salvador, Nicaragua, Costa Rica et Panamal et le Mexique ? Quand on fait référence à l'Amérique latine, de manière générale, on souligne des éléments d'identité qui sont loin d'être exacts. Qu'entend-on exactement par «Amérique latine » d'un point de vue géographique? L'ensemble des pays d'Amérique du sud et d'Amérique centrale? Certes, mais le Mexique appartient, selon les géographes, à l'Amérique du Nord. Peut-être, pour simplifier, doit-on se contenter d'englober sous cette dénomination les nations situées au sud du Río Bravo lle fleuve qui marque la frontière entre les États-Unis et le Mexique)? Mais alors il faudrait admettre que la Guyane et le Surinam font partie de l'Amérique «latine». L'unité culturelle, aussi, peut être mise en cause. Sont-elles latines ces Amériques formées par des peuples noirs issus du continent africain? Est-elle latine la société du Guatemala dont $55 \%$ de la population descend des Mayas et parle des langues indigènes? Est-il Latin le Belize ${ }^{02}$ ? Il parait évident que les disparités entre pays, tant au regard de leur taille que de leur potentiel économique ou culturel, ne favorisent pas une réelle conscience unitaire.

Néanmoins, au sein de l'Amérique latine (le Mexique et l'Amérique centrale compris), il est possible d'identifier plusieurs traits communs, comme son rôle périphérique. Il s'agit d'une Amérique marquée par la colonisation espagnole et portugaise. Ces pays ont les mêmes problèmes de développement économique. Ils ont aussi, une appartenance culturelle à l'Occident, car les civilisations précolombiennes n'ont pas résisté aux envahisseurs qui ont imposé leurs langues, leurs valeurs et leur religion ${ }^{03}$. Ces histoires parallèles ont forgé des réalités qui, sans être forcément identiques, ont des points communs qui les distinguent d'autres régions du monde. Dans ces pays, par exemple, à une industrialisation tardive et faiblement autonome, a correspondu une urbanisation fortement concentrée et inégale ${ }^{04}$. La violence, l'insécurité ${ }^{05}$ et une importante économie informelle sont des éléments que l'on retrouve et qui unissent les sociétés latino-américaines.

Le Mexique et l'Amérique centrale ont un autre point commun : leur tradition juridique. Le droit, en Amérique latine, est fortement inspiré du droit europée ${ }^{06}$. On retrouve donc, à l'échelle régionale, un droit présentant une forte dose d'idéalisme, de paternalisme, de légalisme, de formalisme et qui

01 L'Amérique centrale est la région géographique du continent américain située entre l’Amérique du Nord et l'Amérique du Sud. Elle est entourée par l'océan Pacifique et l'océan Atlantique. Politiquement, il y a sept pays : le Guatemala, le Belize, le Honduras, le Salvador, le Nicaragua, le Costa Rica et le Panama.

02 A. Rouquié, Amérique latine. Introduction à l'Extrême-Orient, Éditions du Seuil, Paris, 1987, p. 15.

03 Ibidem, pp. 20-21.

04 J.P. Deler « Un espace marqué par la métropolisation », Problèmes d’Amérique Latine, n¹4, 1994, pp. 41-44.

05 F. Martin Cubel, El Estado de la seguridad en América Latina, Documento Opinión, Instituto Español de Estudios Estratégicos, Enero de 2016, http://www.ieee.es/Galerias/fichero/docs opinion/2016/DIEEE001 2016 Seguridad AmericaLatina FernandoMartinCubel.pdf

06 R. David, "L'originalité des droits de l'Amérique latine », in R. David, Le droit comparé, droits d'hier, droits de demain, Economia, Paris, 1982, p. 166. 
parvient difficilement à pénétrer la société, empêchant la consolidation de l'État de Droit ${ }^{07}$. En ce qui concerne le droit du travail, celui-ci a connu un développement significatif et s'est concrétisé sous la forme de codes du travail remarquables. Pourtant, il reste toujours un grand problème de mise en application ${ }^{08}$.

On constate aussi que le Mexique et les pays d'Amérique centrale partagent une même histoire coloniale. Ils ont une culture occidentale et des cultures indiennes syncrétiques. S'il est vrai que le Mexique et l'Amérique Centrale connaissent une période de modernisation, il est aussi vrai que la marginalisation d'une grande partie de la population l'oblige à se réfugier dans l'économie informelle, phénomène structurel et non conjoncturel (I), qui se manifeste d'une manière complexe dans les pays analysés (II).

\section{I - Emploi informel : phénomène structurel et complexe}

L'emploi informel, au Mexique et en Amérique centrale, constitue un phénomène permanent et structurel (A). Un nombre important mais aussi une grande diversité d'emplois sont affectés, ce qui rend parfois difficile l'identification des types de travail concernés (B).

\section{A - Un phénomène structurel}

$\mathrm{Au}$ Mexique et en Amérique Centrale, les crises économiques répétitives, le chômage, le sous-emploi, les bas salaires de l'économie formelle, ainsi que la décentralisation de la production vers le secteur informel -en tant que stratégie pour réduire les coûts de production-, ont contribué à la naissance, à la croissance et au développement de l'économie informelle.

L'accroissement de l'économie informelle s'explique par le besoin de survie, le désir d'indépendance, la flexibilité, la précarisation du marché du travail et même par le désir de se voir assurer des revenus plus élevés que ceux pratiqués au sein du secteur formel ${ }^{09}$. En 2013, l'emploi informel représentait 46,8\% de l'emploi non agricole en Amérique latine, un taux en recul de $3.3 \%$ par rapport à celui de 2009 , alors qu'il atteignait $50,1 \%{ }^{10}$. En 2014, l'emploi informel représentait 42\% de l'emploi non agricole au Panama ${ }^{11}$. En 2013 le taux d'informalité au Costa Rica était de 30,7\%, 60,1\% au Salvador, $72,8 \%$ au Honduras, $73,6 \%$ au Guatemala et $82 \%$ au Nicaragua (population sans sécurité sociale).

07 K.L. Karst, K.S Rosenn, Law and Development in Latin America. A Case Book, 1975, p. 65.

08 N. Álvarez, et al. “Derechos laborales en Centroamérica y Panamá”, Informe Estados de la Región, 2008; M. García Villegas, “Ineficacia del derecho y cultura del incumplimiento de reglas en América Latina", in C. Rodríguez Garavito, (coord.), El derecho en América Latina: un mapa para el pensamiento jurídico del siglo XXI, Buenos Aires, Siglo Veintiuno Editores, 2011, pp. 160-184; J. Weller, (comp.), «Elementos para el perfeccionamiento de la institucionalidad laboral de Panamá: El contexto regional », in Perfeccionamiento de la institucionalidad laboral en Panamá, 2009, pp. 33 et s.

09 Selon l'Enquête nationale de micro-entreprises de l'INEGI, 5\% de la population se trouvait dans l'économie informelle parce qu'elle ne trouvait pas de travail, tandis que $95 \%$ avaient basculé soit pour rejoindre des activités familiales soit parce qu'ils avaient trouvé là un meilleur niveau de revenu. http:// www.beta.inegi.org. $\mathrm{mx} /$ proyectos/enchogares/modulos/enamin/2012/.

10 R. Guerra, A. Joslyn, El trabajo informal en Panamá, Encuesta de Propósitos Múltiples. Marzo 2014, Ministerio de Economía y Finanzas, Panamá, 2015, p. 8; CEPAL/OIT, Coyuntura laboral en América Latina y el Caribe Formalización del empleo y distribución de los ingresos laborales, Octubre de 2014, n¹1, p. 15.

11 Fundación del Trabajo, Desafíos para la formalización de la economía informal en Panamá. Visión desde el mercado laboral, Boletín Electrónico, n³, julio de 2014, p.7, http://www.funtrab.org.pa/uploads/ boletin 3 - julio 2014.pdf. 


\section{Emploo INFormel au Mexique et en Amérique CEntrale}

\section{Amérique Latine (14 pays) : Taux d'emploi informel non agricole par catégorie professionnelle, $2013^{12}$}

\begin{tabular}{|l|c|}
\hline & Taux d'informalité \\
\hline Secteur public & $15,9 \%$ \\
\hline Secteur privé (salariés et employeurs) & $32,9 \%$ \\
\hline Entreprises de 1 á 10 salariés & $58,6 \%$ \\
\hline Entreprises comptant plus de 10 travailleurs & $14,4 \%$ \\
\hline Indépendants & $82,3 \%$ \\
\hline Service domestique & $77,5 \%$ \\
\hline Travailleurs familial auxiliaire & $100,0 \%$ \\
\hline Total & $\mathbf{4 6 , 8} \%$ \\
\hline
\end{tabular}

Source : SIALC de l'OIT sur la base d'enquêtes auprès des ménages.

Note: Employés âgés de 15 ans et plus dans les zones urbaines.

L’emploi informel est généralement de faible productivité et de mauvaise qualité ${ }^{3}$. Les travailleurs informels sont particulièrement exposés aux risques et à la vulnérabilité, en particulier : la maladie ; les accidents du travail et les maladies professionnelles; la perte de revenus due au handicap; le licenciement et la vieillesse. En outre, les travailleurs n'ont pas la possibilité de faire valoir leurs droits en matière d'heures de travail, de rémunération des heures supplémentaires, de salaire minimum, de congés payés et d'adhésion syndicale, entre autres ${ }^{14}$.

\section{B - La complexité de l'emploi informel}

L'OIT, au fil des ans, a élaboré une définition de l'économie informelle qui tourne autour de la notion d'emploi. Cela constitue un point de départ afin de connaître la diversité des situations relevant de l'économie informelle des pays analysés : travailleurs, travailleurs indépendants, employeurs informels, petites entreprises informelles et travailleurs informels travaillant pour une société formelle.

À l'occasion d'une mission du BIT au Kenya, en 1972, visant l'établissement d'une stratégie globale pour l'emploi, la notion de secteur informel apparaît pour la première fois. Ses caractéristiques principales sont alors : la facilité d'accès à l'activité ; le recours aux ressources locales; la propriété familiale des entreprises; l'échelle restreinte des opérations; les techniques à forte intensité de main-d'œuvre et dûment adaptées; les qualifications acquises en dehors du système scolaire officiel et les marchés échappant à tous les règlements et ouverts à la concurrence ${ }^{15}$.

12 L. Deelen, (éd.), Políticas para la formalización de las micro y pequeñas empresas en América Latina. Experiencias, avances y desafíos, Santiago de chile, Organización Internacional del Trabajo, 2015, p. 12.

13 Fundación del Trabajo, Desafíos para la formalización..., op. cit., p.1.

14 L. Deelen (editora), Políticas para la formalización..., op. cit., p. 27.

15 P.-M. Roy, et P. Bodson, « Peut-on formaliser le secteur informel ? », in P. Bodson, P.M.Roy, Politiques d'appui au secteur informel dans les pays en développement, Economica, Paris, 1995, p. 4. 
Cette démarche est d'une importance particulière aux fins du droit du travail, car on y retrouve pour la première fois une définition de nature fonctionnelle, centrée autour de l'emploi. Pour l'OIT, le secteur informel représente : « un ensemble d'unités produisant des biens ou des services en vue principalement de créer des emplois et des revenus pour les personnes concernées. Ces unités, ayant un faible niveau d'organisation, opèrent à petite échelle et de manière spécifique, avec peu ou pas de division entre le travail et le capital en tant que facteurs de production. Les relations d'emploi - lorsqu'elles existent - sont surtout fondées sur l'emploi occasionnel, les liens de parenté ou les relations personnelles et sociales plutôt que sur des accords contractuels comportant des garanties en bonne et due forme $»^{16}$.

L'OIT a récemment considéré plus approprié de se référer à l'économie informelle plutôt qu'à un secteur informel parce que: « ...cette expression recouvre une diversité considérable de travailleurs, d'entreprises et d'entrepreneurs qui sont dotés de caractéristiques identifiables et connaissent des désavantages et des problèmes dont l'intensité varie suivant le contexte, national, urbain ou rural. L'expression « économie informelle » est préférable à celle de « secteur informel » parce que les travailleurs et les entreprises dont il est question ne relèvent pas d'un secteur unique d'activité économique mais de plusieurs. En revanche, elle tend à minimiser l'importance des liens, des zones grises et des interdépendances qui existent entre activités formelles et activités informelles. L'expression « économie informelle » fait référence à toutes les activités économiques de travailleurs et d'unités économiques qui ne sont pas couverts - en vertu de la législation ou de la pratique - par des dispositions formelles. Ces activités n'entrent pas dans le champ d'application de la loi, ce qui signifie que ces travailleurs et unités opèrent en marge de la loi; ou bien ils ne sont pas couverts dans la pratique, ce qui signifie que la loi ne leur est pas appliquée alors même qu'ils opèrent dans le cadre de la loi ; ou bien encore la loi n'est pas respectée parce qu'elle est inadaptée, contraignante ou qu'elle impose des charges excessives. Le BIT doit tenir compte dans ses travaux des difficultés conceptuelles liées à cette immense diversité ${ }^{17}$.

Bien que l'intention initiale soit la création d'emplois pour pouvoir s'assurer d'un revenu, très fréquemment l'informalité résulte du fait que les salariés, travaillant dans des entreprises formelles, ne sont pas déclarés ou sont embauchés comme travailleurs indépendants là leur compte). Il peut aussi y avoir des cas d'entreprises formelles qui sous-traitent des activités à d'autres entreprises qui, à leur tour, n'ont pas inscrit leurs travailleurs à la sécurité sociale et ne respectent pas les règles applicables au travail salarié. Pour la Recommandation n²04 de l'OIT, les termes « économie informelle »:

«...al désignent toutes les activités économiques des travailleurs et des unités économiques qui - en droit ou en pratique - ne sont pas couvertes ou sont insuffisamment couvertes par des dispositions formelles; b) ne désignent pas les activités illicites, en particulier la fourniture de services ou la production, la vente, la possession ou la consommation de biens interdites par la loi, y compris la production et le trafic illicites de stupéfiants, la fabrication et le trafic illicites d'armes à feu, la traite des personnes et le blanchiment d'argent, tels que les définissent les conventions internationales pertinentes ${ }^{18}$.

16 B.I.T., Quinzième Conférence internationale des statisticiens du travail. Rapport de la conférence, janvier 1993. http://www.ilo.org/wcmsp5/groups/public/---dgreports/---stat/documents/normativeinstrument/ wcms_087485.pdf.)

17 B.I.T, Résolution concernant le travail décent et l'économie informelle. Conférence générale de l'Organisation internationale du Travail, réunie en sa 90e session, 2002.

18 Conférence Internationale du Travail, Recommandation 204 concernant la transition de l'économie informelle vers l'économie formelle, adoptée par la Conférence à sa 104e session, Genève, 12 juin 2015. 


\section{Emploi informel au Mexique et en Amérlque centrale}

Il conviendrait de prendre en compte également, suivant l'approche préconisée par la Recommandation $n^{\circ} 204$, le fait que le travail informel peut être observé dans tous les secteurs de l'économie, aussi bien dans les espaces publics que dans les espaces privés ${ }^{19}$. Cette Recommandation renvoie à la Résolution concernant le travail décent et l'économie informelle ainsi qu'à la définition fonctionnelle de 1993 de l'OIT, dans la mesure où elle exclut, elle aussi, les activités illicites du périmètre de l'économie informelle. La Recommandation stipule en effet que les unités économiques de l'économie informelle comprennent :

a) les unités qui emploient de la main-d'œuvre ;

b) les unités détenues par des particuliers travaillant à leur propre compte, soit seuls, soit avec le concours de travailleurs familiaux non rémunérés et,

c) les coopératives et les unités de l'économie sociale et solidaire.

De surcroît, la Recommandation permet de reconnaître différents types d'acteurs dans l'économie informelle. Elle s'appliquerait ainsi :

«...à tous les travailleurs et à toutes les unités économiques de l'économie informelle, comprenant les entreprises, les entrepreneurs et les ménages, en particulier :

a) aux personnes opérant dans l'économie informelle qui détiennent et administrent des unités économiques, y compris : les travailleurs à leur propre compte, les employeurs, les membres des coopératives et des unités de l'économie sociale et solidaire ;

b) aux travailleurs familiaux non rémunérés, qu'ils travaillent dans des unités économiques de l'économie formelle ou de l'économie informelle;

c) aux salariés ayant des emplois informels au sein des entreprises formelles ou des unités économiques de l'économie informelle, ou travaillant pour elles, notamment dans le cadre de la sous-traitance et des chaînes d'approvisionnement, ou en tant que travailleurs domestiques rémunérés employés par des ménages;

d) aux travailleurs dont la relation de travail n’est pas reconnue ou pas réglementée ».

La Recommandation $n^{\circ} 204$ se réfère aussi bien aux unités de production qu'aux travailleurs, dans la mesure où il est parfois difficile de les distinguer les uns des autres. Tel est le cas lorsque, par exemple, un travailleur exerce une activité à la fois comme travailleur indépendant ou comme employeur. Les catégories retenues par la Recommandation constituent un cadre de référence susceptible de nous permettre d'étudier l'emploi informel au Mexique et en Amérique centrale, et cela même si les frontières entre ces catégories ne sont pas toujours claires.

19 Au Mexique, l'Administration publique compte un nombre important d'employés embauchés sur la base d'honoraires, qui devraient avoir une relation de travail formelle (35\%). Source: ENOE 2015. 


\section{II - Types d'emplois informels au Mexique et en Amérique centrale}

L'un des avantages de la Recommandation n²04 de l'OIT est d'identifier les types d'emplois présents dans l'économie informelle, que l'on rencontre, pour la plupart, au Mexique et en Amérique centrale $(A)^{20}$. Ces emplois ont fait l'objet de politiques publiques qui n'ont pas eu le succès désiré quant à la réduction de l'informalité (B).

\section{A - Travailleurs, travailleurs indépendants, employeurs, entreprises et ménages}

Dans le cas du Mexique et de l'Amérique Centrale, on peut envisager le travail dans l'économie informelle autour de quatre groupes : celui des travailleurs ly compris le travail familial, le travail non rémunéré, le travail domestique et le travail agricole) (1) ; celui des travailleurs indépendants (2); celui des entreprises informelles ou des employeurs utilisant des travailleurs informels (3) et, enfin, celui des entreprises formelles ou employeurs qui utilisent l'informalité pour développer des relations de travail masquées $(4)^{21}$.

Au Panama, par exemple, la part des travailleurs, travailleurs indépendants et employeurs au sein de l'économie informelle est la suivante en 2013 : 1,4\% de travailleurs auxiliaires, 6,8\% d'employeurs, $16,8 \%$ de salariés et $75,1 \%$ de travailleurs autonomes. Cette dernière catégorie est celle qui connaît les conditions de travail les plus précaires, la plus grande productivité et les écarts de revenus les plus importants ${ }^{22}$.

\section{1 - Les travailleurs}

Les « travailleurs » exercent leur activité souvent dans des conditions dangereuses du point de vue de la sécurité et de la santé au travail. Ils ont des revenus et des horaires de travail irréguliers ${ }^{23}$. Bien qu'ils bénéficient de certains droits, ceux-ci sont inférieurs aux standards établis par la législation du travail. De même, ils n'ont pas de couverture sociale, ce qui les rend vulnérables. On peut aussi trouver parmi eux des travailleurs " déguisés » en travailleurs indépendants ${ }^{24}$. La Résolution concernant le travail décent et l'économie informelle souligne ainsi que " les travailleurs de l'économie informelle peuvent se caractériser par des degrés divers de dépendance et de vulnérabilité » (conclusion $n^{\circ}$ 9).

Au Mexique, d'après l'Instituto Nacional de Estadística y Geografía (INEGI) la population occupée de manière informelle, qui comprend toutes les formes d'emploi informel, totalise 29,5 millions de personnes. Le secteur informel, pour l'INEGI, se compose :

- de la population occupée dans les unités économiques non agricoles exploitées sans comptabilité et fonctionnant, sans être constituée en société, à partir des ressources du ménage ou de la personne qui dirige l'activité ;

20 F. Delgado Jiménez, "El empleo informal en Costa Rica : características de los ocupados y sus puestos de trabajo", Revista de ciencias económicas, Vol. 31, n² 2, 2013, pp. 35-51; R. Guerra, A. Joslyn, « El trabajo informal en Panamá... », op. cit., pp. 9 y 10; J. Mezzera, "Apuntes sobre la heterogeneidad en los mercados de trabajo de América Latina" in D. Carboneto, et. al., El sector informal en los países andinos, ILDIS/CEPESIU, Ecuador, 1995, p. 64.

21 F. Delgado Jiménez, “El empleo informal..., op. cit., pp.35-51.

22 Fundación del Trabajo, Desafíos para la formalización..., op. cit., p. 8.

23 OIT, Cartilla pedagógica como herramienta para facilitar procesos organizativos y formativos con trabajadores de la economía informal, Consejo Sindical Unitario de América Central y Caribe, 2014, p. 6.

24 F. Delgado Jiménez, “El empleo informal en ..., op. cit., p. 41. 


\section{EMPLOI INFORMEL AU MEXIQUE ET EN AMÉRIQUE CENTRALE}

- du travail domestique ;

- du travail agricole non protégé et

- des travailleurs occupés dans des unités économiques formelles sans couverture sociale ${ }^{25}$.

Dans l'économie informelle la composante féminine est forte. Les femmes sont généralement des salariées qui occupent des rangs inférieurs et sont parfois victimes de discrimination, comme c'est également le cas dans le secteur forme ${ }^{26}$. L'emploi informel dans le service domestique représentait 5\% de l'emploi total en Amérique latine en $2013^{27}$.

Au Panama, 30\% des hommes travaillent dans le secteur informel. Cette proportion est réduite à $23 \%$ s'agissant des femmes. Mais cette asymétrie est compensée par une incidence plus élevée de l'informalité féminine dans les services domestiques, avec $8 \%$ des femmes employées, contre $1 \%$ pour les hommes ${ }^{28}$. Les femmes jouent donc un rôle important dans l'économie informelle au Mexique et dans l'Amérique centrale (a). On peut en dire autant des travailleurs ruraux (b) et de certains travailleurs vulnérables dans l'informalité du travail (c).

\section{a - Travail domestique}

En Amérique latine, les caractéristiques de l'emploi domestique, qui le rapprochent de l'activité informelle, comme l'insuffisance de revenu, le manque de protection contre les risques (santé, vieillesse, maladie et chômage) et la forte incidence de la pauvreté sont largement déterminés par l'absence ou les limites de la couverture des travailleurs domestiques par les législations du travail29.

Au Mexique, le travail domestique est réglementé par la Loi Fédérale du travail (LFT) qui, en lui reconnaissant une nature spéciale, ne lui accorde pas une protection complète. A titre d'exemple, l'employeur des travailleurs domestiques, n'est pas obligé de les assurer au régime de sécurité sociale. Par conséquent, même lorsque leurs droits au travail sont respectés, ils restent informels puisque l'accès à la couverture sociale reste facultatif. Selon les données de l'Encuesta Nacional de Ocupación y Empleo (ENOE, 2017), il y'aurait actuellement 2285538 travailleurs domestiques ${ }^{30}$.

Dans le cas des services domestiques panaméens, la grande majorité des travailleurs sont des femmes. Elles ont un emploi informel ou sont simplement privées de couverture sociale. La principale raison de cette exclusion est probablement due au fait que le taux de la contribution sociale (incluant les cotisations patronales et salariées) augmente les coûts salariaux de $22 \%$, ce qui pourrait expliquer la réticence de nombreux ménages ayant des revenus moyens à s'y soumettre,

25 INEGI, Encuenta Nacional de Ocupación y Empleo. Cifras del segundo trimestre de 2017, 14 de agosto de 2017, http://www.inegi.org.mx/saladeprensa/boletines/2017/enoe ie/enoe ie2017 08.pdf.

26 C. Rakowski, "Desventaja multiplicada. La mujer del sector informal”, Nueva Sociedad, n90, pp. 134-146; M. Pollack, ¿ Feminización del sector informal en América latina y el Caribe?, Naciones Unidas, Chile, 1993, p. 59; D. Castillo Fernández, "La mujer en el sector informal ¿De qué hablamos?", Acta sociológica, UNAM, México, $n^{\circ} 7$, pp. 9-21

27 CEPAL/OIT, Coyuntura laboral en América Latina..., op. cit., p. 15.

28 Fundación del Trabajo, Desafíos para la formalización..., op. cit., p.1.

29 M. Cebollada Gay, "Propuesta de política pública para la formalización del trabajo del hogar remunerado en México", en OIT, Protección social del trabajo doméstico Tendencias y estadísticas, OIT, 2017; M. G. Loyo y M.D. Velásquez, "Aspectos jurídicos y económicos del trabajo doméstico remunerado en América Latina", in M. E. Valenzuela, y C. Mora, Trabajo doméstico: un largo camino hacia el trabajo decente Santiago, Oficina Internacional del Trabajo, 2009, p. 69; DHUCA, Investigación Reconociendo el Trabajo Doméstico Remunerado en El Salvador, UCA, OIT, 2015, p. 162; OIT, La legislación salvadoreña a la luz del Convenio sobre el Trabajo Decente para las trabajadoras y los trabajadores domésticos, nº189, 2011, 0IT, 2015, pp. 57 y ss.

30 INEGI, Encuesta Nacional de Ocupación y Empleo..., op. cit. 
sans pour autant justifier leur conduite. Dans d'autres cas, len particulier chez les ménages à revenu élevél, il peut exister un problème culturel et un manque de sensibilisation sur l'impact du respect de cette obligation ${ }^{31}$.

\section{b - Travailleurs agricoles}

Dans de nombreux pays, il existe un secteur rural informel composé de petites exploitations, d'activités traditionnelles artisanales et commerciales, ainsi que d'agriculture de subsistance. Il a été souligné que, dans de nombreux pays, la plupart des activités du secteur informel sont réalisées dans les zones rurales ${ }^{32}$. Au Mexique, l'INEGI estime que ce chiffre est de 5,9 millions de personnes ${ }^{33}$.

\section{c- Travailleurs vulnérables}

Les enfants, les femmes ${ }^{34}$, les jeunes ${ }^{35}$, les migrants ${ }^{36}$, les populations autochtones et les personnes âgées constituent le « gros » des travailleurs vulnérables ${ }^{37}$. Les femmes occupent souvent des emplois improductifs et non rémunérés, ce qui s'explique apparemment par la rigidité du marché du travail ainsi que par des facteurs culturels qui les empêchent d'accéder à des emplois plus productifs. Dans le cadre de l'emploi informel, il y a une sur-représentation des femmes dans la catégorie des travailleurs familiaux sans rémunération et une sousreprésentation chez les employeurs ${ }^{38}$.

\section{2 - Travailleurs indépendants}

Le travailleur indépendant est celui qui gère son unité économique, reproduisant en partie des formes archaïques de capitalisme, tant dans l'organisation que dans les méthodes de production ${ }^{39}$. Le travailleur indépendant informel est celui qui gère une entreprise produisant des biens ou des services qui:

- ne constitue pas une personne morale autre que le ménage ou les membres du ménage auxquels elle appartient ;

- ne dispose pas d'un mécanisme de comptabilité ;

- entretient la confusion entre ses activités productives et son patrimoine propre (puisqu'il peut être salarié de sa propre unité de production dès lors qu'un seul travailleur est requis).

- ne déclare pas les revenus ni les flux de capitaux de l'entreprise et sa qualité de propriétaire ou de salarié de sa propre entreprise.

31 Fundación del Trabajo, Desafíos para la formalización..., op. cit., p. 9.

32 J. Charmes, "Approches comptables, statistiques et économiques du secteur informel, AMIRA lamélioration des méthodes d'investigation et de recherche appliqués au développement) brochure nº61, pp. 5-53.

33 INEGI, Encuesta Nacional de Ocupación y Empleo..., op. cit.

34 A. Seligson, "Las mujeres en el sector informal costarricense : Causas del éxito", Anuario de Estudios Centroamericanos, n²4, 1998, pp. 21-46.

35 OIT, Formalizando la informalidad juvenil: experiencias innovadoras en América Latina y el Caribe, Lima, OIT, Oficina Regional para América Latina y el Caribe, 2015, p. 71.

36 L. Paniagua Arguedas, “Situación socio-laboral de la población nicaragüense en Costa Rica”, Revista de Ciencias Sociales, n¹17-118, 2007, pp. 57-72.

37 A. Brenes Narváez y F. Cruz Rivera, “Determinantes de la informalidad en Nicaragua”, Revista de Economía y Finanzas BCN, 2016, pp. 124 y ss.

38 L. Deelen (Editora), Políticas para la formalización de las micro..., op. cit., p.31.

39 J. Charmes, “Débat actuel sur le secteur informel”, Revue Tiers Monde, n¹12, 1987, pp. 855-875. 


\section{Emploo informel au Mexique et en AmérlQue centrale}

Le travailleur indépendant couvre tous les besoins de sa propre entreprise. Il est une sorte de chef d'orchestre qui, malgré le fait qu'il ne dispose en général pas de formation, doit malgré tout déployer toute son ingéniosité pour mener à bien son activité. Cette image plus ou moins positive ne peut pas être généralisée à tous travailleurs indépendants. Parmi eux, il y a aussi des travailleurs de rue qui exercent des activités qui leur permettent tout juste de survivre jour après jour.

Les travailleurs indépendants peuvent aussi exercer leurs activités dans des conditions dangereuses et se trouver dans une situation de réelle vulnérabilité, à l'instar des salariés informels. Leur situation est parfois extrême, lorsque leurs activités sont de l'ordre de la survie ou lorsqu'elles relèvent d'une logique de développement. Elles devraient en principe, dans cette dernière hypothèse, leur permettre d'accumuler du capital et de l'investir, afin de progresser économiquement en donnant à leur activité un certain potentiel commercial ${ }^{40}$. Cependant, étant donné qu'ils évoluent dans l'économie informelle, leur accès au crédit demeure très incertain.

En 2013, l'emploi informel dans le secteur informel - qui comprend principalement les autoentrepreneurs et les microentreprises informelles non enregistrées - représentait en Amérique latine près des $2 / 3$ de l'emploi informel et $30,7 \%$ de l'emploi total non agricole. Les taux d'informalité les plus élevés, en 2013 , ont été observés chez les travailleurs indépendants $(82,7 \%)$, suivis par les travailleurs domestiques $(79,1 \%)$ et ceux des micro entreprises comptant moins de 10 travailleurs ${ }^{41}$.

\section{3 - Petites entreprises informelles ou employeurs informels utilisant des travailleurs informels}

Une entreprise individuelle informelle peut être créée par un seul membre du ménage, plusieurs membres du même ménage ou par différents ménages ${ }^{42}$. L'entreprise informelle est détenue et exploitée par son propriétaire, seul ou en association avec des membres du ménage ou de ménages différents. Elle emploie des travailleurs permanents len plus des travailleurs familiaux non rémunérés). Leur activité est principalement une activité de services, de fabrication, de réparation ${ }^{43}$ ou de fourniture de services ${ }^{44}$. Dans les entreprises informelles, on a remarqué qu'il existe deux extrêmes : il peut s'agir d'une simple activité de subsistance ou bien cette activité peut s'inscrire dans une ambition plus large, offrant aux entrepreneurs informels l'opportunité de se développer, de s'enrichir ${ }^{45}$. Une proportion élevée des emplois générés par ce type d'unité est informelle. En 2013, le taux d'emploi informel chez les indépendants était de $82 \%$ et parmi les petites entreprises de $59 \%$. Selon le BIT, toujours en 2013, les travailleurs indépendants et les entreprises ayant un effectif de 10 travailleurs au plus représentent $70 \%$ de l'emploi informel total dans la région ${ }^{46}$.

Les travailleurs indépendants et les propriétaires d'une entreprise familiale exercent généralement deux rôles. Tout en étant responsables de la gestion de l'entreprise, ils exercent en même temps des tâches qui auraient pu, en principe, être confiées à un salarié. Les activités productives d'une entreprise familiale procurent deux types de revenu : une rémunération attachée à la qualité de chef d'entreprise et une rémunération pour le travail effectivement fourni. C'est la raison pour laquelle ce revenu est généralement décrit comme mixte ${ }^{47}$.

40 Fundación del Trabajo, Desafíos para la formalización..., op. cit., p. 8.

41 CEPAL/OIT, Coyuntura laboral en América..., op. cit., p. 15.

42 OIT, Quinzième Conférence internationale..., op. cit., p. 62 ; F. Wils, The informal sector in greater Sao Paulo: A discussion of concepts and criteria, and a description of its characteristics, Institute of Social Studies (Working Papers), The Hague-The Netherlands, 1991, p. 21.

43 OIT, Quinzième Conférence internationale..., op. cit., p. 63.

44 R. Guerra, A. Joslyn, En trabajo informal en Panamá..., op. cit., p. 12.

45 Fundación del Trabajo, Desafíos para la formalización..., op. cit, p. 8.

46 L. Deelen, (ed.), Políticas para la formalización..., op. cit.

47 OIT, Quinzième Conférence internationale..., op. cit. p. 18. 
Au niveau microéconomique, parmi les principaux déterminants qui influent sur la décision des petites entreprises de fonctionner de manière informelle, on trouve ${ }^{48}$ :

- la faible productivité des micro et petites entreprises qui ne permettrait pas de s'acquitter des coûts de la formalité.

- La complexité des procédures d'enregistrement des entreprises, le poids des charges et de la fiscalité, ainsi qu'une réglementation inadéquate.

- L'attractivité de l'informalité comme option de flexibilité et d'indépendance, par opposition aux rares avantages de la formalité.

- Un contrôle réduit et une faible possibilité d'être sanctionné.

L'entreprise informelle rencontre de multiples problèmes comme, par exemple, des restrictions pour pouvoir solliciter des financements via des entités financières formelles. L'informalité implique des restrictions dans l'accès au marché, la participation aux marchés publics et la publicité. Ces restrictions peuvent aussi concerner la circulation et le transport des biens et des produits. L'exploitation dans le secteur informel implique aussi des obstacles pour établir des contrats avec des travailleurs, des fournisseurs et des clients, au-delà de la famille et des amis. Les entreprises informelles sont également susceptibles d'être extorquées par des fonctionnaires corrompus et sont exclues des services fournis par les différents organismes publics dédiés au développement productif ${ }^{49}$.

\section{4 - Entreprises formelles ou employeurs formels utilisant des travailleurs informels}

Dans l'économie informelle, interviennent aussi des entreprises formelles qui vont se fournir auprès d'entreprises ou de travailleurs indépendants informels ${ }^{50}$. En 2013, en Amérique latine, l'emploi informel dans le secteur formel représentait $11,7 \%$ du total des emplois non agricoles, en raison du non-respect du cadre réglementaire, du manque de connaissance de la loi ou de faiblesses institutionnelles ${ }^{51}$.

La décentralisation de la production à laquelle se livrent les grandes entreprises, qui ont un recours systématique à la sous-traitance, s'est également traduite par l'augmentation de l'informalité. Les travailleurs sont ainsi embauchés par des entreprises sous-traitantes (recourant à de fausses relations indépendantes). II n'est pas surprenant qu'au Mexique, la soustraitance ait été l'objet d'une réglementation en 2012, afin d'assurer que la société sous-traitante ou donneuse d'ordre respecte bien ses obligations juridiques en matière de sécurité sociale et de droit du travail.

Les mesures de formalisation de l'emploi adoptées par les gouvernements visent la formalisation des micro et petites entreprises et soulignent également la formalisation du travail indépendant où les niveaux d'informalité sont assez élevés. En ce qui concerne la formalisation des entreprises informelles, cela implique également la formalisation des emplois qu'elles génèrent ${ }^{52}$.

48 L. Deelen (Editora), Políticas para la formalización..., op. cit.

49 Ídem.

50 R. Guerra, A. Joslyn, En trabajo informal en Panamá..., op. cit., pp. 9 y10; INEGI, Encuesta Nacional de Ocupación y Empleo.... op. cit.

51 CEPAL/OIT, Coyuntura laboral..., op. cit., p. 2.

52 D. Martínez, Políticas Activas de Mercado de Trabajo para generar empleo y mejorar la calidad de los empleos en América Latina, Santiago, Comisión Económica para América Latina y el Caribe, 2016, p. 30. 


\section{Emplol informel au Mexique et en Amérique centrale}

\section{B - Stratégies de formalisation de l'emploi}

Au Mexique et en Amérique centrale, il existe un grand nombre de programmes pour formaliser les emplois (1). Cependant, les résultats sont difficiles à apprécier positivement, il semble que ces politiques n'aient pas eu l'effectivité escomptée et n'aient pas réduit fondamentalement le nombre d'emplois informels (2).

\section{1 - Programmes de formalisation de l'emploi.}

Poussons Ensemble (PS) est un plan mexicain d'avantages sociaux, fiscaux et de crédits immobiliers pour lutter contre l'informalité. Le programme a été créé pour les travailleurs indépendants et les petites entreprises informelles. Ceux qui en bénéficient peuvent ${ }^{53}$ :

- Obtenir un crédit immobilier. Le contribuable qui verse des contributions à INFONAVIT (Institut du Fonds national pour la promotion de l'habitat des travailleurs) pendant quatre mois (s'il a un employeur) ou sept mois (s'il n'a pas de relation de travail), peut accéder à un prêt. Le prêt peut être utilisé pour refinancer des prêts hypothécaires existants. En plus, le gouvernement subventionnera une partie des paiements hypothécaires.

- Accéder à la sécurité sociale. Avec le paiement d'un quota préférentiel réduit pendant 10 ans, il est possible accéder à l'Instituto Mexicano de Seguridad Social (IMSS) pour une couverture maladie, maternité, invalidité et vieillesse, etc.

- Soutenir les entrepreneurs. Accès à un soutien économique de 4000 pesos mexicains lenviron 230 euros) pour améliorer l'engagement commercial. Grâce à Bansefi (Banque d'épargne nationale et services financiers) 2000 pesos sont accordés pour un compte bancaire débiteur et 2000 pesos supplémentaires peuvent être obtenus pour soutenir l'investissement.

- Financement des entreprises. Accès à des crédits de 20.000 à 300.000 pesos. Les prêts sont destinés principalement aux entreprises ayant plus de 2 ans d'activité. La durée de remboursement varie de 24 à 60 mois selon la destination des ressources.

- Crédit à la consommation. Accès au crédit du Fond National pour la Consommation des Travailleurs. L'argent peut être utilisé pour l'achat d'appareils électroniques, de services touristiques, de meubles, de soins médicaux, entre autres.

Le Programme de Formalisation de l'Emploi (PFE), également développé par le Mexique, favorise l'enregistrement des travailleurs informels à l'Institut Mexicain de la Sécurité Sociale (IMSS) et encourage l'assurance volontaire des travailleurs indépendants à l'IMSS ${ }^{54}$. De plus, étant donné que de nombreux travailleurs informels exercent leur activité comme travailleurs indépendants pour le compte d'organismes publics fédéraux ou locaux, le PFE cherche à promouvoir leur inscription à l'IMSS, en invitant ces institutions à les affilier. De même, le Programme vise à formaliser l'emploi de tous les travailleurs des fournisseurs et entrepreneurs privés ayant des contrats avec l'administration publique fédérale et locale ${ }^{55}$.

Au Mexique il existe des programmes spécifiques au sein des différents États fédérés en vue de la formalisation de l'emploi. On peut citer à titre d'exemple Hidalgo, Querétaro et Chihuahua, qui ont fait des efforts de formalisation au profit des travailleurs indépendants des transports publics. Dans

53 Gobierno de México, ¿Qué hacemos?, 2 de junio de 2015: www.crezcamosjuntos.gob.mx.

54 Au Mexique, il existe une assurance obligatoire et une autre volontaire á l'IMSS.

55 Programa de Promoción de la formalización en América Latina y el Caribe, El empleo informal en México: situación actual, políticas y desafíos, FORLAC-OIT-Oficina regional para América Latina y el Caribe, 2014, p.10. 
l'État d'Hidalgo, on aurait ainsi formalisé 400 travailleurs. Dans le même registre, il a été prévu de régulariser la situation de 1,534 travailleurs municipaux informels. À Querétaro, le Réseau Q,- qui vise à améliorer l'efficacité des transports publics a permis de formaliser 2000 conducteurs déjà inscrits à l'IMSS. À Chihuahua, le nouveau système de transports publics, Vivebús, en place depuis 2013, s'est traduit par la formalisation de1056 emplois de conducteurs alors qu'aucun d'entre eux n'avait de contrat et que plus de $80 \%$ n'avaient pas de couverture sociale ${ }^{56}$.

Au Costa Rica, les petites entreprises bénéficient d'un taux préférentiel d'impôt sur le revenu lloi 7092) et d'une exonération de la taxe sur les personnes morales (loi 9024 de l'impôt sur les personnes morales). Pour profiter de cette dernière exemption, la société doit être enregistrée au registre de petites entreprises du Ministère de l'économie. Cette inscription ne fait que délivrer des certifications aux entreprises qui sont à jour de leurs différentes obligations, renforçant ainsi la formalisation ${ }^{57}$.

Les pays d'Amérique centrale ont mis en place une série de stratégies réglementaires, administratives et de développement, axées avant tout sur le développement économique des petites entreprises ${ }^{58}$. De même, ces pays ont fait d'énormes efforts pour promouvoir le développement productif des petites entreprises qui créent une grande partie des emplois, dont beaucoup de faible qualité et dans des conditions d'informalité. Dans certains pays, si des progrès ont été réalisés, les niveaux d'informalité restent supérieurs à la moyenne pour l'Amérique latine. L'informalité, dans les pays considérés, a une ampleur et une hétérogénéité considérables. Toutefois ils partagent au moins une caractéristique commune: un niveau élevé de travail autonome ou indépendant et un travail familial non rémunéré qui représente près de $50 \%$ des effectifs. La formalité lappréciée au regard du bénéfice d'une assurance maladie) est presque inexistante sur ces segments du marché. L'autre moitié de la main-d'œuvre se compose de relations de travail avec les travailleurs rémunérés ou salariés, dont le degré de formalisation croît en fonction de la taille de l'entreprise. Le rôle de la micro finance reste dominant parmi les politiques micro économiques les plus utilisées dans ces pays. Des politiques ou des actions spécifiques sont également nécessaires dans les secteurs dominés par les entreprises indépendantes et familiales, où se trouve concentrée la majeure partie du secteur informel len particulier dans le secteur rural) ${ }^{59}$.

\section{2 - Résultats des programmes de formalisation de l'emploi}

L'emploi informel se caractérise par une juxtaposition et une confusion de fonctions. La même personne peut en même temps et à différents stades être salarié, travailleur indépendant ou employeur informel. À cette juxtaposition de rôles, il convient d'associer les employeurs formels qui utilisent l'informalité par le biais du recours à la sous-traitance frauduleuse ou par l'embauche de faux indépendants.

Les deux programmes mexicains mentionnés ci-dessus (PS et PFE) présentent plusieurs inconvénients. D'abord, le type d'emplois ou les travailleurs informels visés par ces programmes ne sont pas identifiés. Deuxièmement, ils ne sont pas soumis à une évaluation permanente. Troisièmement, le gouvernement fédéral et les gouvernements des États n'ont pas fait d'effort pour formaliser tous les travailleurs occupés par l'administration publique, qui n'ont accès ni à la sécurité sociale ni aux

56 Ibidem, p. 8

57 FORLAC/OIT, Políticas para la formalización de las micro y pequeñas empresas en Costa Rica, FORLAC/ OIT, 2014, p. 14

58 L. Deelen, (ed.), Políticas para la formalización..., op. cit.

59 J. Chacaltana, Experiencias de formalización empresarial y laboral en Centroamérica: un análisis comparativo en Guatemala, Honduras y Nicaragua, Santiago de Chile, Naciones Unidas, CEPAL, 2009, p. 73. 
avantages sociaux. Bien que le taux d'emploi informel soit passé de $60 \%$ à $57 \%$ en quatre ans, il n'est pas sûr que cette baisse soit due aux programmes fédéraux ou étatiques en faveur de la formalisation de l'emploi. En effet, des changements dans les politiques budgétaires ont forcé de nombreuses personnes à régulariser leur situation financière et par conséquent leur situation professionnelle.

La plupart des évaluations sur l'emploi informel ne couvrent pas l'ensemble des politiques de formalisation. Elles cherchent simplement à mesurer l'impact des programmes spécifiques dans les pays concernés ou dans quelques régions en particulier. La Comisión Económica para América Latina yel Caribe (CEPAL), auteur d'un rapport global sur les résultats des politiques de formalisation de l'emploi, souligne - en citant une étude de l'IFC de la Banque mondiale dans plusieurs pays de la région - que pour les entrepreneurs ayant opté pour la formalité, les principales motivations sont : le fait d'éviter les amendes pour violation de la loi ou les pots de vin. Les avantages n'apparaissent qu'en quatrième position, à savoir le fait d'obtenir de nouveaux clients ou des marchés pour les entreprises. Cela semble indiquer que les avantages de la formalité ne constituent pas une évidence pour les petites entreprises: "Ceci est important parce que, si les avantages sont très faibles, on peut continuer à réduire les coûts de la formalité, celle-cin'en sera pas plus attrayante $»^{60}$.

En analysant les résultats des différents types de mesures introduites en Amérique latine, susceptibles d'encourager la formalisation de l'emploi informel, - telles que la déclaration des travailleurs à la sécurité sociale, la simplification des procédures d'enregistrement des entreprises, la régularisation de la propriété informelle, le monotributo ${ }^{61}$ et la création d'un guichet unique; le renforcement institutionnel des inspections du travail et des impôts, ainsi que l'impact des politiques microéconomiques pour formaliser l'emploi informel - il a été observé, que toutes ces mesures ne donnent pas nécessairement les résultats escomptés. Les avantages produits ne compensent pas les inconvénients découlant des coûts élevés de la formalité ${ }^{62}$. Par contre, les faibles salaires reçus dans le secteur formel, le respect limité de la réglementation du travail et le faible niveau de formation professionnelle de nombreux travailleurs des pays analysés sont une invitation permanente à les faire travailler dans l'informalitéb3. Finalement, en ce qui concerne les faux indépendants, il est également vital que l'État prenne les mesures nécessaires pour obliger les entreprises à respecter les exigences légales.

60 D. Martínez, Políticas Activas de ..., op. cit., pp. 30 y ss.

61 Le Monotributo est un régime fiscal intégré et simplifié qui consiste à payer une redevance mensuelle fixe pour concentrer en une seule taxe les obligations en matière: d'impôt sur le revenu; de taxe sur la valeur ajoutée (TVA) ; de contributions au régime de pension publique du système intégré de retraite (SIJP), de contribution au système national d'assurance maladie.

62 D. Martínez, Políticas Activas de ..., op. cit., pp. 55 y ss.

63 J. Weller (comp.), « Elementos para el perfeccionamiento de la institucionalidad... », op. cit., pp. 133 y ss.

\section{ALFREDO SÁNCHEZ-CASTAÑEDA}

\section{Professeur à l'Institut de Recherche Juridique de l'UNAM.}

Thèmes de recherche : Travailleurs précaires, Emploi des jeunes, Travailleurs non salariés, Droits fondamentaux au travail, Inégalités salariales. Parmi ses publications : “El artículo 123 constitucional y el derecho al trabajo: ¿Un necesario cambio de paradigma?", en Suprema Corte de Justicia, La herencia del constitucionalismo social mexicano y sus desafíos, México, SCJN, 2017, pp. 213-300.

(en coll.) P. Kurczyn, 'Mexico' International Encyclopaedia of Laws: Labour Law and Industrial Relations, R. Blanpain (ed.); Alphen aan den Rijin, NL; Kluwer Law International, 2016, 174 pp. 